\title{
Discussion: Machine learning to inform tunnelling operations: recent advances and future trends
}

Brian B. Sheil

Department of Engineering Science, University of Oxford, Oxford, UK (Orcid:0000-0002-1462-1401) (corresponding author:

brian.sheil@eng.ox.ac.uk)

\section{Stephen K. Suryasentana}

Department of Engineering Science, University of Oxford, Oxford, UK Michael A. Mooney

Department of Civil and Environmental Engineering, Colorado School of Mines, Golden, CO, USA

\section{Contribution by Bryan A. McCabe and Kevin G. O'Dwyer}

The authors have compiled an excellent review of machine learning approaches and their potential to support decision making in tunnelling operations; this is valuable and timely given the copious data generated nowadays on tunnelling projects. The application of non-probabilistic machine learning to tunnelling inherently assumes complete confidence in the data used. However, since machine learning is not yet used for predictive purposes on commercial tunnelling projects, and since the data generated are rarely analysed retrospectively by tunnelling practitioners, the quality control systems necessary to ensure the reliability of these data are not in place. This inadequacy has yet to be exposed in the academic literature because, as pointed out by the authors, most machine learning methodologies have only been validated against a single case history.

In the case of pipe-jacking/microtunnelling, with which the contributors are familiar, jacks are used at multiple points in the process (including at the launch shaft, intermediate jacking points and the steering cylinders immediately behind the tunnel-boring machine (TBM) face. While Sterling (2020) states that pipejacking forces are typically known with reasonable accuracy; the load cells at these points of force application are rarely subjected to regular calibration and therefore the reliability of data generated must be questioned. Equally, injected lubricant volumes are measured using flowmeters, which are not routinely calibrated, and despite the availability of modern navigation systems, a trundle wheel (which sits on top of the pipe in the launch shaft) is still commonly used to assess jacked length in shorter drives.

An example from the authors' files is used to highlight data quality problems. Force versus jacked distance relationships for a pipe-jacking drives in clay are illustrated in Figure 12. The frictional force (along the pipe string) shown in Figure 12 is derived in two ways:

(i) Total jacking load minus the load in the steering cylinders (where the latter is measured, it is assumed to correspond to the force at the TBM face).

\author{
Hehua Zhu \\ Department of Geotechnical Engineering, College of Civil Engineering, \\ Tongji University, Shanghai, China \\ Bryan A. McCabe \\ Senior Lecturer, Civil Engineering, School of Engineering, National \\ University of Ireland, Galway, Ireland \\ Kevin G. O'Dwyer \\ Research Student, Civil Engineering, School of Engineering, National \\ University of Ireland, Galway, Ireland
}

(ii) Approximated from a straight line joining minima in the jacking load against jacked distance data (Pellet-Beaucour and Kastner, 2002). This method of interpretation is typically used in the absence of measurements enabling frictional load and load at TBM face to be separated.

It can be seen from Figure 12 that the estimates in (i) and (ii) differ significantly, and that the data in (i) imply negative values of shaft friction early in the drive, which are not credible. Therefore, the frictional forces for these drives cannot be interpreted with certainty. The authors strongly suspect that such data quality problems are widespread in the tunnelling industry, and that the value of such data for on-site predictions is currently limited. Therefore, quality control measures (such as frequent calibration, maintenance, replacement of measurement equipment) need to be implemented by tunnelling operators to ensure that the data are of sufficient quality to unlock the potential of both backanalysis (e.g. McCabe et al., 2012; O'Dwyer et al., 2020) and machine learning (Sheil et al., 2020) in contributing to our understanding of tunnelling processes.

\section{Authors' reply}

The authors thank the contributors for their valuable insights into current challenges associated with practical implementation of the data analysis techniques covered in Sheil et al. (2020). Machine learning involves distilling models from observations to make inferences and, as such, is inextricably linked with uncertainty. Uncertainty is often broadly categorised as either 'epistemic' (relating to the model itself) or 'aleatoric' (relating to the stochasticity of observations). The contributors highlight the prominence of the latter in the tunnelling industry, which is most commonly attributable to signal noise, infrequent sensor calibration and temperature effects.

Classical regression techniques, such as linear regression and artificial neural networks, assume certain and precise measurement of the system to be modelled. As noted by the contributors, this is a gross simplification for many practical applications. For this reason, there is a long history of 'fuzzy' 


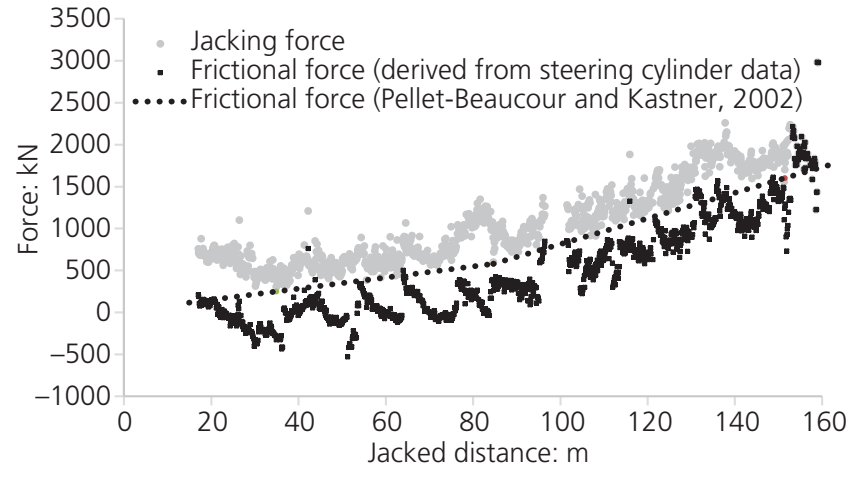

Figure 12. Pipe-jacking force versus jacked distance data used to highlight uncertainties in frictional force for a drive in clay

methods, such as adaptive neuro-fuzzy inference systems and fuzzy logic, applied to tunnelling in the literature. This stems from their ability to incorporate human experience and deal with imprecise and noisy data typical of construction monitoring.

The authors welcome the contributors' endorsement for continued application of machine learning methods employing probabilistic frameworks such as Bayesian networks and Gaussian process regression. In a Bayesian setting, epistemic uncertainty, reflected by the prior probability, is updated by the likelihood and derived from an aleatory sampling model. Uncertainty is therefore included explicitly in the modelling, providing a more principled framework for treating signal artefacts and dealing with noisy and missing data. Given the significant cost of mistakes in this industry, robust treatment and consideration of uncertainty is a vital underpinning element of the decision-making process (Sheil, 2020).

While these methods present exciting opportunities for the tunnelling sector, the authors agree that more high-quality data is needed for further validation of these algorithms, such as generalising to different construction techniques and sites, to promote more widespread industry take-up.

\section{REFERENCES}

McCabe BA, Orr TLL, Reilly CC and Curran BG (2012) Settlement trough parameters for tunnels in Irish glacial tills. Tunnelling and Underground Space Technology 27(1): 1-12.

O'Dwyer KG, McCabe BA and Sheil BB (2020) Interpretation of pipejacking and lubrication records for drives in silty soil. Underground Space 5(3): 199-209.

Pellet-Beaucour AL and Kastner R (2002) Experimental and analytical study of friction forces during microtunnelling operations. Tunnelling and Underground Space Technology 17(1): 83-97.

Sheil B (2020) Prediction of microtunnelling jacking forces using a probabilistic observational approach. Tunnelling and Underground Space Technology 109: 103749

Sheil BB, Suryasentana SK, Mooney MA and Zhu H (2020) Machine learning to inform tunnelling operations: recent advances and future trends. Proceedings of the Institution of Civil Engineers - Smart Infrastructure and Construction, https://doi.org/10.1680/jsmic.20.00011. Sterling R (2020) Developments and research directions in pipe-jacking and micro-tunnelling. Underground Space 5(1): 1-19.

\section{How can you contribute?}

To discuss this paper, please email up to 500 words to the editor at journals@ice.org.uk. Your contribution will be forwarded to the author(s) for a reply and, if considered appropriate by the editorial board, it will be published as discussion in a future issue of the journal.

Proceedings journals rely entirely on contributions from the civil engineering profession (and allied disciplines). Information about how to submit your paper online is available at www.icevirtuallibrary.com/page/authors, where you will also find detailed author guidelines. 